\title{
Left ventricular opacification during selective intracoronary injection of echocardiographic contrast in patients with hypertrophic cardiomyopathy
}

\author{
P M Elliott, S J Brecker, W J McKenna
}

\begin{tabular}{|c|c|c|c|c|c|c|}
\hline \multicolumn{2}{|c|}{$\begin{array}{l}\text { Department of } \\
\text { Cardiological } \\
\text { Sciences, St George's } \\
\text { Hospital Medical } \\
\text { School, Cranmer } \\
\text { Terrace, London } \\
\text { SW17 0RE, UK } \\
\text { P M Elliott } \\
\text { S J Brecker } \\
\text { W J McKenna }\end{array}$} & \multicolumn{5}{|c|}{$\begin{array}{l}\text { cardiomyopathy. Increasingly, echocar- } \\
\text { diographic contrast agents are used before } \\
\text { alcohol injection to identify the perfusion } \\
\text { bed of the septal perforator vessels. This } \\
\text { study describes the unexpected opacifica- } \\
\text { tion of the left ventricular cavity in three }\end{array}$} \\
\hline \multicolumn{2}{|c|}{$\begin{array}{l}\text { Correspondence to: } \\
\text { Dr Elliott } \\
\text { email: pelliott@sghms.ac.uk } \\
\text { Accepted } 23 \text { November } 1999\end{array}$} & \multicolumn{5}{|c|}{$\begin{array}{l}\text { of five consecutive patients following } \\
\text { selective injection of the first septal perfo- } \\
\text { rator with Optison. This case study dem- } \\
\text { onstrates that direct communication }\end{array}$} \\
\hline \multicolumn{7}{|c|}{ Table 1 Demographic data } \\
\hline Patient Age (years) & hest pain & $Y H A$ & Syncope & $I V S(\mathrm{~mm})$ & $\operatorname{Grad}(\mathrm{mm} \mathrm{Hg})$ & $L V o p$ \\
\hline & & & & & & \\
\hline & & & & & & \\
\hline & & & & & & \\
\hline & & & & & & \\
\hline & & & & & & \\
\hline
\end{tabular}

NYHA, New York Heart Association functional class; IVS, interventricular septum; Grad, peak resting left ventricular outflow tract gradient; LV, left ventricular.

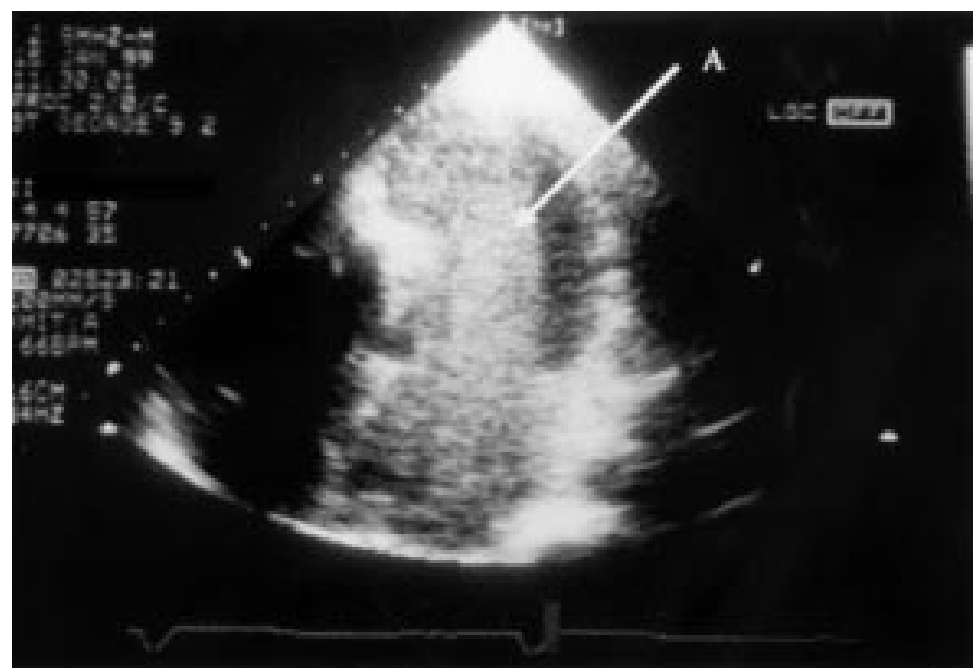

Figure 1 Apical four chamber view of the left ventricle following injection of $0.5 \mathrm{ml}$ of Optison into the first septal perforator vessel. There is dense opacification of the anterior septum, and almost immediate opacification of the left ventricular cavity $(A)$. between the first septal perforator vessel and the left ventricle is common, an observation that may have considerable relevance to the technique of alcohol septal reduction.

(Heart 2000;83:e7)

Keywords: hypertrophic cardiomyopathy; ventricular obstruction; septal alcohol ablation

\section{Case study}

Five consecutive patients (all women, mean (SD) age 43 (2.5) years, range 41-47) with obstructive hypertrophic cardiomyopathy were studied (table 1). All had asymmetric septal hypertrophy (mean wall thickness 20 (3) $\mathrm{mm}$, range 16-25) and resting left ventricular outflow tract obstruction (mean gradient 89 (23) $\mathrm{mm} \mathrm{Hg}$, range 64-121) on continuous wave Doppler, in association with systolic anterior motion of the mitral valve. All five had refractory chest pain and/or dyspnoea, despite medical treatment with $\beta$ blockers and disopyramide. Coronary angiography was uncomplicated in all five patients. There was no evidence of left ventricular opacification, or coronary artery fistulae during selective left and right coronary injections in any patient.

The first major septal branch of the left anterior descending artery was catheterised using a $2 \mathrm{~mm}$ coaxial angioplasty balloon catheter (15 mm NC Bandit; Boston Scientific, Minnesota, USA). Following balloon inflation, angiographic contrast medium (Urograffin; Schering AG, Berlin, Germany) was injected through the central lumen to ensure that there was no spill-back into the left anterior descending artery. With the balloon still inflated, $0.5 \mathrm{ml}$ of Optison (Mallinckrodt Medical $\mathrm{GmbH}$, Hennef, Germany) was injected via the central lumen of the angioplasty balloon while performing transthoracic echocardiography (2.5 MHz transducer, Hewlett Packard Sonos 1000) from the apical four chamber view.

Opacification of the basal interventricular septum was observed in all five patients. In one patient (patient 1) opacification extended down to the left ventricular apex. In three of the five patients (table 1), septal opacification was 


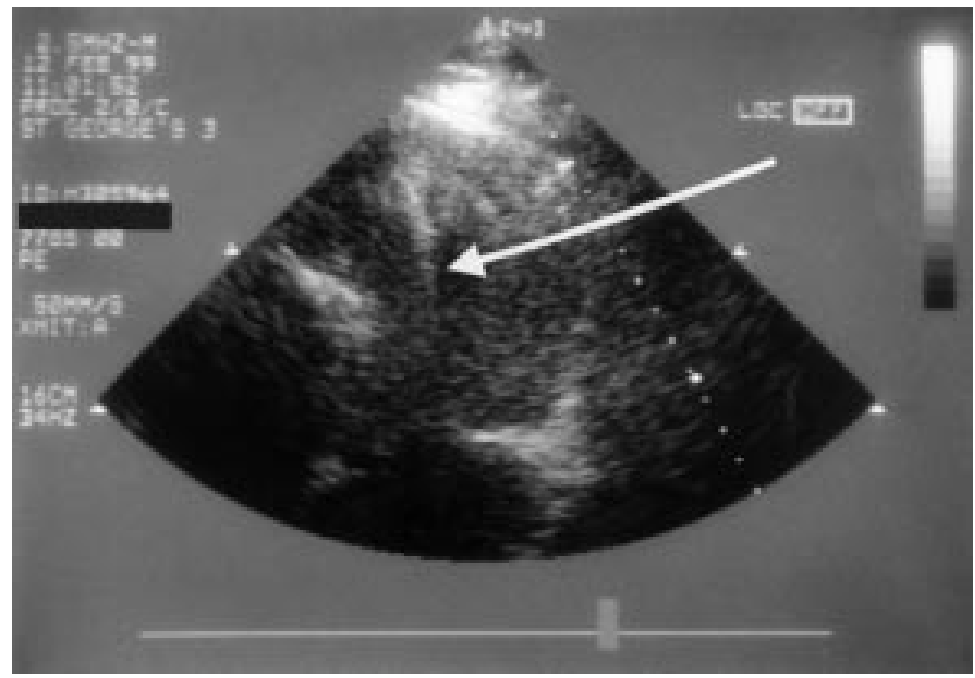

Figure 2 Apical four chamber view demonstrating a "jet" of echo contrast agent emerging from the left ventricular apex (arrow) following injection of Optison into the first septal perforator branch of the left anterior descending artery.

followed immediately by the appearance of echo contrast in the left ventricular cavity (figs 1 and 2). In view of the extensive perfusion bed of the first septal artery in patient 1 , alcohol was not injected and the patient subsequently underwent successful myotomy-myectomy. In the four remaining patients with left ventricular opacification, $2-3 \mathrm{ml}$ of desiccated alcohol were injected through the central lumen of the inflated angioplasty catheter. In patient 4 , there was a brief run of ventricular tachycardia that was terminated successfully by overdrive pacing. In patient 5 , there was transient complete heart block lasting for 24 hours.

\section{Discussion}

Selective injection of alcohol into the first septal perforator branch of the left anterior descending coronary artery is an alternative to septal myotomy-myectomy in selected patients with hypertrophic cardiomyopathy and severe left ventricular outflow tract obstruction. ${ }^{1-4}$ In some centres, new generation intravenous echocardiographic bubble contrast media have been used to demarcate the perfusion bed of the septal perforator vessel before injection of alcohol. This helps to ensure that only the relevant vessel is occluded, and prevents unwanted infarction of larger areas of myocardium. This report demonstrates that new generation echo contrast media can also detect direct communications between the first septal perforator vessel and the left ventricle. The presence of such communications has potentially important implications for the technique of non-surgical septal reduction in patients with hypertrophic cardiomyopathy.

CORONARY VENTRICULAR “FISTULAE"

Angiographically demonstrable coronary artery fistulae are rare, the most common occurring between the right coronary artery and the right atrium. Coronary ventricular connections are even rarer, accounting for only $2 \%$ of all coronary artery fistulae. A very small number of coronary ventricular fistulae have been reported in association with hypertrophic cardiomyopathy, mostly in patients with apical hypertrophy. ${ }^{5}$ The five cases described in this report had neither angiographic evidence for coronary ventricular fistulae nor hypertrophy confined to the left ventricular apex, and it is likely that the ventricular opacification seen following Optison injection represents drainage via small coronary ventricular channels.

NORMAL MYOCARDIAL VENOUS DRAINAGE

Myocardial venous drainage channels were first described by Vieussens and Thebesius in the 18 th century, ${ }^{67}$ and then later by Wearns and colleagues. ${ }^{8}$ However, it was only in the 20 th century that the functional importance of arterioventricular communications between the coronary arteries and the left ventricular cavities was first investigated. Using ${ }^{131} \mathrm{I}$ labelled albumin, Moir and colleagues demonstrated that approximately $80 \%$ of septal perforator blood flow in dogs (approximately 13\% of total left coronary artery blood flow) drains directly into the right ventricle, ${ }^{9}$ thereby escaping coronary sinus drainage. In a subsequent dog study, ${ }^{10}$ Moir and colleagues demonstrated that direct drainage into the left ventricle usually occurs via the circumflex artery and, in particular, via the left anterior atrial branch. Direct communication between the first septal perforator vessel and the left ventricle was demonstrable in only one of 10 animals. Data on coronary ventricular drainage channels in humans are more limited. Cornel et al demonstrated that left ventricular opacification following left main stem injection of echo contrast occurred in 10 of 29 patients undergoing routine coronary angiography, two of whom had hypertrophic cardiomyopathy. ${ }^{11}$ The route by which contrast entered the ventricle could not be determined, but outflow jets from the myocardium were observed from the apex, anterolateral wall and interventricular septum. Interestingly, the earliest opacification occurred in the two patients with hypertrophic cardiomyopathy.

CLINICAL RELEVANCE OF CORONARY ARTERY-VENTRICULAR COMMUNICATIONS

Several groups have reported their experience with echo contrast before septal alcohol ablation, but none has reported ventricular opacification following selective injection of the septal perforator vessel. ${ }^{1-4}$ It is possible that the high frequency of ventricular opacification in this study is serendipitous, but a more probable explanation is that it reflects our use of undiluted contrast medium, and perhaps the physical characteristics of the specific agent used in this study.

A concern arising from our observation is that inadvertent leakage of alcohol into the left ventricular cavity through coronary ventricular channels may be a potential hazard to patients. The risk of a systemic effect is probably low as the actual volume of blood that enters the ventricle via the first septal vessel is very small, and thus any alcohol that flows straight through is likely to be diluted very rapidly. However, the risk of recirculation of small amounts of alcohol into the epicardial coronary vessels is unknown, and it is possible that some of the 
procedure related complications such as ventricular arrhythmia and heart block may relate to the presence of arterioventricular channels.

CONCLUSION

A lack of data in humans means that we cannot say whether communications between the septal perforator vessel and the left ventricle are more common in patients with hypertrophic cardiomyopathy. Even if they are a normal phenomenon, their presence may be of considerable relevance to outcome of septal alcohol ablation.

We gratefully acknowledge the assistance and advice of Professor Robert Anderson (Imperial College School of Medicine, National Heart and Lung Institute, London), Professor Nigel Brown (Department of Anatomy and Developmental Biology, Stown (Department of Anatomy and Developmental Biology, St George's Hospital Medical S
Knight in preparing this paper.

1 Sigwart U. Non-surgical myocardial reduction for hypertrophic obstructive cardiomyopathy. Lancet 1995;346:21114.
2 Knight C, Kurbaan AS, Seggewiss H, et al. Non-surgical reduction for hypertrophic obstructive cardiomyopathy: outcome in the first series of patients. Circulation 1997;95: 2075-81.

3 Seggewiss H, Gleichmann U, Faber L, et al. Percutaneous transluminal septal myocardial ablation in hypertrophic obstructive cardiomyopathy: acute results and 3-month follow-up in 25 patients. 7 Am Coll Cardiol 1998;31:252-8.

4 Nagueh SF, Lakkis NM, He ZX, et al. Role of myocardial contrast echocardiography during nonsurgical septal reduction therapy for hypertrophic obstructive cardiomyopathy. $f$ Am Coll Cardiol 1998;32:225-9.

5 Wong CK, Lau CP, Cheung CH. Coronary artery-left ventricular fistula with apical hypertrophic cardiomyopathy. Eur Heart f 1991;12:283-5.

6 Vieussens R. Nouvellas découvertes sur le coeur. Paris, 1706.

7 Thebesius AC. Disputatio medica de circulo sanguinis in corde. Lugduni Batavorum, 1708.

8 Wearn JT, Mettier SR, Klump TG, et al. The nature of the vascular communications between the coronary arteries and the chambers of the heart. Am Heart f 1933;9:143.

9 Moir TW, Eckstein RW, Driscol TE. Thebesian vein drainage of the septal artery. Circ Res 1963;12:212-19.

10 Moir TW, Driscol TE, Eckstein RW. Thebesian drainage in the left heart of the dog. Circ Res 1964;14:245-9.

11 Cornel JH, Ten Cate FJ, Serruys PW. Myocardial contrast echocardiography can depict thebesian vein outflow in humans. Am Heart f 1992;123:1373-4. 